\title{
Meine Erfahrungen bei Lungentuberkulose mit Jod-Methylenblau und Kupferpräparaten.
}

Von

\author{
Prof. Dr. E. Meissen (Hohenhonnef).
}

Wenn wir die Tuberkulose als eine Infektionskrankheit bezeichnen, weil wir ihren Erreger genau kennen, so sagen wir etwas heutzutage Selbstverständliches. Das Wesen dieser Volkskrankheit erschöpfen wir aber damit nicht ganz: Weit mehr als bei den gewöhnlichen Infektionskrankheiten treten, zumal bei der Lungentuberkulose, die Verhältnisse der Abstammung, des Körperbaues, des Berufes und der Lebensweise mitbestimmend, vielfach sogar entscheidend, hervor. Dazu kommt, dass so ziemlich alle offenen Tuberkulosen alsbald zu komplizierten Sekundärinfektionen Veranlassung geben. Hieraus ergeben sich bei dieser Krankheit für die direkte Bekämpfung des Erregers offenbar mancherlei besondere Schwierigkeiten. Der indirekte Weg, die Einwirkung auf den kranken Organismus, durch Ausschaltung von Schädlichkeiten und durch Benutzung aller Bedingungen, die zur Hebung der natürlichen Heilkräfte geeignet sind, hat sich deshalb bisher als der erfolgreichere erwiesen. Derartige hygienisch-diätetische Massnahmen werden stets die unentbehrliche Grundlage der Behandlung der Tuberkulose bleiben.

Die arzneiliche Heilung der Tuberkulose hat aber von jeher, lange bevor man den Erreger kannte, das Sinnen und Sehnen der Menschen beschäftigt, ohne dass bis heute das Ziel erreicht worden wäre. Auch die grossen auf das Tuberkulin und die TuberkuloseHeilsera gesetzten Erwartungen haben sich leider nur zu einem bescheidenen Teil erfüllt: Es ist ein umstrittenes Problem geblieben, 
wie wir die Heilkraft des Tuberkulins regelmässig und grofahrlos zur Geltung bringen können. Noch unsicherer ist die Heilwirkung der Sera. Die Chemotherapie der Tuberkulose, etwa nach dem glänzenden Vorbilde des Salvarsans, hat deshalb neues Interesse gewonnen. Die Empirie hat dies Gebiet bisher fast ganz beherrscht. Unzählige Mittel sind empfohlen und wieder verlassen worden: Keines hat dauernde Anerkennung gefunden. Exakt und methodisch durchgeführte Versuche liegen freilich kaum vor: Die wissenschaftliche Arbeit der beiden letzten Jahrzehnte ist fast ausschliesslich der Erforschung des Tuberkulins gewidmet worden: ein vielleicht notwendiges, aber etwas einseitiges Vorgehen.

Im Juni 1911 machte mir der leider inzwischen verstorbens Prof. Finkler die Mitteilung, dass er gemeinsam mit seiner Mitarbeiterin Gräfin Linden chemische Substanzen gefunden habe, die im Tierversuch eine deutliche Heilwirkung bei Tuberkulose zeigten, und fragte, ob ich die Versuche bei meinen Lungenkranken weiterführen wolle. Ich gestehe, dass ich der Sache zunächst einige Skepsis entgegenbrachte. Nachdem ich mich aber durch wiederholte Besuche und Besprechungen im Hygienischen Institut zu Bonn mit Finkler und der Gräfin Linden von den überraschenden Ergebnissen der Tierversuche überzeugt hatte, bin ich der ehrenden Aufforderung des befreundeten Gelehrten gern gefolgt.

Es waren zwei verschiedene, an sich längst bekannte Chemikalien, die mir übergeben wurden: Das eine, ein mit Jod substituierter Teerfarbstoff, das Jod-Methylenblau, Tetramethylthioninjodid, eignete sich ohne Schwierigkeit zur subkutanen oder intramuskulären Injektion beim Menschen. Der Farbstoff wurde in wässriger Lösung von 2-3\% benutzt, und davon 2-3 mal wöchentlich $2-5 \mathrm{ccm}$ eingespritzt. Die Injektionen sind so gut wie schmerzlos; die grünlichblaue Verfärbung des Harns hat keine Bedeutung. Das Mittel kann in 1-2\% iger Lösung auch leicht intravenös angewandt werden; die intravenöse Applikation bietet aber keine Vorteile und verursacht gelegentlich Schüttelfröste, die jedenfalls mit einer Wirkung auf die Leukozyten zusammenhängen.

Die andere Substanz, die wasserlösliche Chlorverbindung des Kupfers, das Kupferchlorid, wurde in einer Lösung von 1-20\% mit einem Zusatz von Chlornatrium wöchentlich $1-2 \mathrm{mal} \mathrm{zu} 1 / 2$ bis $1 \mathrm{ccm}$ angewandt. Es zeigte sich, dass dies Mittel auch bei grösster Sorgfalt häufig örtliche Reizung und Entzündung an der Injektionsstelle hervorruft. Wir haben deshalb später mit anderen Kupfersalzen, namentlich mit dem Kupfer-Kaliumtartrat, zuletzt mit 
zusammengesetzten Kupferverbindungen Versuche gemacht, und glauben zurzeit in einer von der Gräfin $\mathrm{L}$ inde $\mathrm{n}$ aufgefundenen komplexen Kupferverbindung des Lezithins ein Präparat gefunden zu haben, das den genannten Missstand kaum noch zeigt, und das voraussichtlich auch noch andere Vorteile bietet. Es soll, sobald möglich, von den B ayerschen Farbenfabriken in den Handel gebracht werden.

Da den Kupferverbindungen im Tierversuch die stärkere Wirkung zukommt, so habe ich vorwiegend mit ihnen gearbeitet. Hierzu veranlassten mich noch einige andere Erwägungen: $\mathrm{Es}$ ist bekannt, dass man eine verderbliche Blattkrankheit des Weinstocks, hervorgerufen durch einen schmarotzenden Pilz, die Peronospora viticola, durch Bespritzen mit einer Lösung von Kupfervitriol und gelöschtem Kalk mit gutem Erfolg bekämpft. Wohlbekannt ist ferner, dass an den Wänden von Aquarien, da, wo man sie mit einer Kupfermünze bestreicht, die lästigen Algen nicht wachsen. Es wird auch behauptet, dass man Hühnereier mit Lösung gewisser Kupfersalze besonders gut konservieren könne, und zwar so, dass sie nach geraumer Zeit sogar noch brütfähig blieben. Das schien darauf hinzudeuten, dass dem Kupfer in geeigneter Form wohl eine elektive Wirkung auf niedere pflanzliche Organismen zukommen dürfte. Auch die chemischen Beziehungen des Kupfers zu gewissen Fetten, also vielleicht auch zu der Wachsschicht des Tuberkelpilzes, gaben zu denken; ich verweise hier auf die Ausführungen der Gräfin Linden.

Vor wenigen Wochen, als wir mit unseren Arbeiten bereits vorläufig fertig waren, entdeckten wir bei der Durchforschung der Literatur das Buch von $\operatorname{Luton}^{1}$ ), aus dem Jahre 1894, das uns allen bis da völlig unbekannt war, und die Anwendung von Kupfersalzen bei Tuberkulose ziemlich ausführlich, aber wenig hritisch empfiehlt. Wir können uns also nicht rühmen, die ersten zu sein, die Kupferpräparate bei Tuberkulose angewandt haben, wir betonen aber dafür unseren Anspruch, die ersten zu sein, die methodische Heilversuche damit anstellten, und zwar so anstellten, wie Robert Koch es 1890 auf dem Kongress zu London gefordert hat: „Dass man nicht den Menschen als Versuchsobjekt wählen, sondern zunächst an Tieren versuchen solle, ob die Beobachtungen, die im Reagenzglase gemacht wurden, auch für den lebenden Tierkörper gelten; erst wenn das Tierexperiment gelungen ist, kann man zur Anwendung am Menschen übergehen."

1) E. Luton, Traitement de la Tuberculose par les sels de Cuivre Paris, G. Steinheil 1894.

Beitrăge zur Klinir der Taberkulose. Bd. XXIII. H. 2. 
So sind wir vorgegangen, und es war gewiss richtig, dass Finkler, nachdem Gräfin Linden die Tierversuche zu einem gewissen Abschluss gebracht hatte, mich zu Versuchen bei Lungentuberkulose aufforderte, und hernach Herm Dr. Strauss mit Versuchen bei äusserer Tuberkulose beauftragte. Diese dreiteilige Arbeitsordnung hat mir die Beschäftigung mit der Sache besonders sympathisch gemacht, und gab uns allen stets wertvolle Anregung. Leider machte rasch zunehmende Krankheit unseren hochverehrten Geheimrat Finkler, der noch im Sommer frisch und fröhlich die auf seine Anregung unternommenen Arbeiten unterstützt hatte, bereits zum Herbst hin mehr und mehr unfähig, uns mit Rat und Tat zur Seite zu stehen. Wir haben das Werk allein weiterführen: müssen, und wünschen, dass es seinem Andenken Ehre bringe.

Meine Versuche begannen im Juli 1911 und beziehen sich auf 47 Lungenkranke, die gleichzeitig die gewöhnliche hygienisch-diätetische Kur im Sanatorium Hohenhonnef durchführten. Ich habe absichtlich vorwiegend mittelschwere Fälle ausgewählt, offene, zum Teil mehr oder weniger fieberhafte Lungentuberkulosen des ausgesprochenen II. Stadiums, manche mit Komplikationen in Larynx oder Pleura. Diese Auswahl wurde gemacht, um ein möglichst sicheres Urteil zu gewinnen: In den leichten Fällen des I. Stadiums ist es schwer zu entscheiden, ob der Erfolg einem Mittel oder der allgemeinen Kur zuzuschreiben ist, und in den ganz schweren Fällen des III. Stadiums darf man auch von einem spezifischen Mittel nicht 'zu viel erwarten. Meine bisherigen Ergebnisse sind folgende:

Der durchschnittliche Kurerfolg der Patienten war recht befriedigend. Ganz schwere, progressive Fälle freilich versagen: Weder der Farbstoff noch das Metallsalz vermochten eine entschiedene Wendung zum Guten herbeizuführen.

Leichte Fälle, deren übrigens nicht viele behandelt wurden, zeigten stets einen glatten Heilungsverlauf, der allerdings nicht mit voller Sicherheit auf die Mittel allein bezogen werden kann.

Den Hauptwert lege ich auf die mittelschweren Fälle, die fast sämtlich mit Kupferpräparaten behandelt wurden. Bei einigen konnte die Kur wegen der Schmerzhaftigkeit der Injektionen oder aus zufälligen Gründen nicht genügend durchgeführt werden. Bei den übrigen war der Verlauf in etwa $80 \%$ recht gut, nach meinem Eindruck wesentlich günstiger als in ähnlichen Fällen, wo nur die allgemeine Kur gebraucht wurde. In einzelnen Fällen hat inich die günstige Wirkung überrascht. So z. B. bei einer jungen Frau, die nach dem Wochenbett an einer recht ausgedehnten, fieberhaften 
Lungentuberkulose erkrankt war, und deshalb keine erfreuliche Prognose bot: Gleichwohl machte sie sich sehr gut, verlor das Fieber, nahm reichlich an Gewicht zu, und erholte sich in jeder Hinsicht. Oder bei einem jungen Manne, der im rechten Ober- und Mittellappen, sowie im Kehlkopf erkrankt war, und seit Monaten fieberte: er hat sich dauernd entfiebert, Lunge und Kehlkopf sind in sehr befriedigenden Zustand, und es ist Heilung zu erwarten. Ausführliche Krankengeschichten will ich hier nicht bringen, da ich sis für eine spätere Arbeit vorbehalte.

Der Erfolg tritt selten nach wenigen Einspritzungen, meist erst nach und nach hervor. Bestehendes Fieber verschwindet langsam; langsam und allmählich bessert sich auch der örtliche Befund, vermindern sich die Rasselgeräusche, und mit ihnen Husten und Auswurf. Ich meine ziemlich sicher beobachtet zu haben, dass der Auswurf rascher bazillenfrei wird als in nicht behandelten Fällen. Die Kur wurde 2-3 Monate und länger fortgesetzt, Schwankungen im Verlauf kommen vor. $\mathrm{Ob}$ und wie weit Dauererfolge erreicht wurden, vermag ich bei der Kürze der Beobachtungszeit nicht zu sagen. Auch weiss ich noch nicht, ob man die Kur besser mit Cnterbrechungen oder ohne Pause durchführt.

In der gewählten Dosierung zeigten die Mittel keine giftigen oder sonst schädlichen Wirkungen, abgesehen von der zuweilen starken Sc̀hmerzhaftigkeit der anfänglichen Kupferpräparate. Deutliche Reaktionen in den Krankheitsherden habe ich mit überzeugender Bestimmtheit nicht beobachtet; doch will ich sie nicht verneinen, da sie gewissen Beobachtungen im Tierversuch entsprechen würden. Fiebersteigerungen, die auf solche Reaktionen bezogen werden könnten, scheinen fast nur dann aufzutreten, wenn ein stärkerer entzündlicher Reiz an der Injektionsstelle entsteht, was ich nur bei Kupferpräparaten gesehen habe. Die Verträglichkeit der Injektionen hängt viel von der Individualität der Patienten ab: die zweckmässigste Injektionsstelle ist die Gegend der Glutäen.

Das langsame Hervortreten des Erfolges kann zu Einwendungen Anlass geben, die ich mir auch selbst gemacht habe. In einigen Fällen zeigte sich eine Wirkung aber doch schneller: z. B. bei einem jungen Mädchen, das ausser einer Spitzeninfiltration einen beginnenden Fungus genu hatte, und seit Monaten dauernd fieberte. Die gewöhnliche Kur und die Behandlung mit einem immobilisierenden Lederverband änderten diesen hartnäckigen Fieberzustand nicht: Das Fieber verschwand aber nach der zweiten oder dritten Kupfereinspritzung endgültig; die Kranke ist zurzeit in sohr gutem Zlustand; idas Knie ist völlig beweglich. 
Die langsame Wirkung entspricht aber auch den anatomischen Verhältnissen bei der chronischen Lungentuberkulose: Es ist klar, dass alle Heilmittel nur allmählich an die meist schon seit Jahren bestehenden, im verschiedenen Entwickelungsstadium befindlichen, zum Teil schlecht vaskularisierten Krankbeitsherde herangelangen können.

Zeigt aber ein Mittel im Tierversuch, dessen Ergebnisse freilich unbedingt feststehen müssen, eine unzweifelhafte Heilwirkung auf den tuberkulösen Prozess, ohne dass der Organismus geschädigt wird, so darf sie auch beim kranken Menschen angenommen werden, und muss sich allmählich geltend machen, wenn wir das Mittel in geeigneter Form und Menge immer wieder zuführen.

Dem Einwand, dass die gebrauchten Dosen doch recht klein gewesen seien, kann man entgegenhalten, dass auch im Tierexperiment keine besonders grossen Mengen Kupfer erforderlich waren, und dass es sich beim Menschen um lange fortgesetzte Kuren handelte, bei denen allerdings auch die allgemeinen Heilungsbedingungen günstig gestaltet waren. Zu dem Versuch einer ,Therapia magna sterilisans", den übrigens schon die örtliche Reizwirkung der anfänglichen Präparate ausschloss, hatte ich aus naheliegenden Gründen, namentlich in Anbetracht der noch wenig klaren Toxikologie unserer Mittel, vorläufig keine Lust.

Es ist natürlich nicht ausgeschlossen, dass durch Vervollkommnung der Präparate und durch andere Bosierung raschere und grössere Wirkungen erreicht werden mögen. Darüber können nur weitere Versuche entscheiden. Wir vermögen den Ärzten noch kein abgeschlossenes Heilverfahren zu übergeben, das mit fest bestimmten Dosen nach bestimmten Vorschriften arbeitet. Vorsichtige Dosierung bei langdauernder Anwendung muss zunächst der Leitsatz sein. Sorgfalt und genaue klinische Beobachtungen sind nötig, wenn auch keine unberechenbaren Wirkungen zu erwarten sind, wie etwa beim Tuberkulin. Wunderdinge, die sich doch niemals erfüllen können, muss man auch nicht erwarten: So einfach, dass man mit einigen schematischen Einspritzungen eine Krankheit, wie die Tuberkulose, heilen würde, wird niemals werden; wir müssen an die eingangs kurz angedeutete Eigenart der Tuberkulose denken! Ausser den Injektionen werden übrigens vielleicht Einreibungen von Salben oder Lösungen der Kupferpräparate in Alkohol und Chloroform therapeutische Verwendung finden. Den innerlichen Gebrauch möchte ich weniger raten, weil der Verdauungsapparat unter keinen Umständen gestört werden soll, und weil die Resorption unsicher ist. 
Meine Herren! Der Weg der arzneilichen Heilung der Tuberkulose ist mit Dormen bestreut, und man muss vorsichtig auf ihm wandeln: Schon mancher hat geglaubt, er liege glatt und offen vor ihm, bis die Dornen stachen. Ich will deshalb für meinen Teil nicht einmal unbedingt ausschliessen, dass die hygienisch-diätetische Gesamtkur und vielleicht eine gewisse Suggestion der Kranken, die sie mit besonderer Zuversicht erfüllte, doch der wesentliche Grund der Erfolge gewesen ist. Ich hoffe nur, dass wir hier wenigstens am Anfang einer Chemotherapie der Tuberkulose stehen, die sich ausgestalten und in der Bekämpfung dieses Leidens bewähren möge: Es ist oft viel versprochen und wenig gehalten worden: Möge es hier einmal umgekehrt werden! 